\author{
EL MAPUCHE HABLADO EN TIRÚA: FONEMAS \\ SEGMENTALES, FONOTAXIS Y COMPARACIÓN \\ CON OTRAS VARIEDADES
}

\author{
MAPUCHE SPOKEN IN TIRUA: PHONEMES AND ALLOPHONES, \\ PHONOTAXIS AND COMPARISON WITH OTHER VARIETIES
}

\title{
GASTÓN SALAMANCA
}

Universidad de Concepción, Chile

gsalaman@udec.cl

\section{ELIZABETH QUINTRILEO}

Universidad de Concepción, Chile equintrileo@udec.cl

\section{RESUMEN}

Este artículo tiene como foco la descripción de los fonemas segmentales y las realizaciones alofónicas del mapuche hablado en Tirúa. Se mencionan, en primer lugar, los estudios fonológicos anteriores realizados sobre la lengua mapuche. En segundo lugar, se detalla la metodología utilizada en la investigación (se mencionan aquí aspectos relativos a la zona donde se recogieron los datos, los informantes que colaboraron en ella y las características más relevantes de las transcripciones y lista léxica utilizadas). Luego, se señala el marco de referencia escogido para el análisis del material fónico (el método descriptivista norteamericano) y se presenta in extenso el inventario de fonemas y realizaciones alofónicas de esta variante del mapuche. En el quinto punto, se indican las características más relevantes de la pauta de combinación de las unidades fonémicas segmentales (se mencionan aquí las características más relevantes de la estructura silábica y de la estructura de la palabra). El sexto punto explicita los casos de fluctuación de fonemas que se detectaron. Por último, y como conclusión de esta investigación, se presenta una síntesis comparativa entre la variante estudiada y las otras variantes dialectales del mapuche.

Palabras claves: Mapuche, fonemas del mapuche, mapuche hablado en Tirúa.

\section{ABSTRACT}

The aim of this article is to provide a description of the segmental phonemes and the allophonic realizations of oral Mapuche in Tirúa. First, earlier phonologic studies on Mapuche are cited. Second, the research methodology is explained (details are given about the area where data were collected, participants, and the most relevant characteristics of the transcriptions and lexical lists used). Third, the theoretical framework selected for the phonic material is provided (the American descriptivist method) and a complete 
inventory of phonemes and allophonic realizations of the Tirúa variant of Mapuche are presented. Then, the most relevant characteristics are indicated of the segmental phonemic model combination (the most relevant characteristics of the syllabic structure and word structure are mentioned here). After that, phoneme fluctuation is described. Last, and as a conclusion of this research work, a comparative synthesis is presented of the Tirúa variant and the other dialectical variants of Mapuche.

Keywords: Mapuche, Mapuche phonemes, Mapuche spoken in Tirúa.

Recibido: 03-01-2008. Aceptado: 24-03-2009.

\section{INTRODUCCIÓN ${ }^{1}$}

Z sta investigación se sitúa en el nivel fonológico. Se estudia aquí la lengua Chablada por la población mapuche de la comuna de Tirúa. Salas (1992a) senala:

Los mapuches llaman a su lengua mapudungu, palabra que significa literalmente "el habla -o la lengua- de este país o de esta tierra", formada sobre las palabras mapu "tierra, país" y dungu "habla, lengua".

Como denominación alternativa es muy usado el sustantivo verbal correspondiente: mapudungun "el hablar de esta tierra" (Salas, 1992a: 57).

El territorio ocupado por los mapuches corresponde al sector chileno ubicado entre el río Bío-Bío y la provincia de Valdivia, en la Región de los Ríos. El pueblo mapuche es el más numeroso del país y uno de los grupos indígenas con mayor cantidad de población en América del Sur. Así, también, la lengua vernácula hablada por este pueblo es una de las más importantes de Sudamérica, motivo por el cual se ha convertido en una de las lenguas más estudiadas. En palabras de Lagos (1981): "Se trata de la lengua vernácula más importante, tanto en el número de hablantes, como por las variedades dialectales que presenta, además de los factores históricos, lo cual ha permitido que sea la lengua indígena más estudiada" (225).

Lenz (1895-1897) distinguió cuatro zonas dialectales mapuches: picunche (mapuche nortino) ${ }^{2}$, moluche (mapuche central), pehuenche (mapuche cordillerano) y huilliche (mapuche sureño). Con algunos matices, esta clasificación es seguida por Croese (1980).

De la clasificación anterior se desprende la clasificación del mapuche de la costa norte como parte del mapuche central, pero no hay evidencias empíricas de esto. Precisamente, la carencia de trabajos realizados en esta zona justifica este tra-

${ }^{1}$ Con leves matices, la introducción y los puntos 2 y 3 de este trabajo se contienen también en Quintrileo y Salamanca (2007).

${ }^{2}$ Variedad extinta, a juicio de Salas (1992a). 
El mapuche hablado en Tirúa: Fonemas segmentales, fonotaxis y comparación... / G. SALAMANCA y E. QuintriLEO

bajo y lo anticipa como un aporte a los estudios dialectales del mapuche en general y de la fonología de esta lengua en particular.

\section{ESTUDIOS SOBRE LA FONOLOGÍA DEL MAPUDUNGUN ${ }^{3}$}

Como se señala en Salamanca (2001), los primeros trabajos sobre la lengua mapuche fueron realizados por misioneros jesuitas, entre los siglos XVII y XVIII, labor que fue continuada por los capuchinos bávaros, quienes arribaron por primera vez a Chile en 1895. El más destacado de ellos fue Fray Félix José de Augusta, cuya lucidez e intuición lingüísticas llevan a decir a Salas (1992b) que

Aunque es posible que los métodos modernos de enseñanza/aprendizaje de lenguas sean más eficaces que el buen viejo y seguro enfoque gramatical, lo cierto es que hasta hoy, las tres grandes obras del P. Félix de Augusta [Gramática Araucana, Lecturas Araucanas y Diccionario Araucano] forman el único programa compacto y bien organizado disponible para el estudio del mapuche (Salas, 1992b: 483).

Con la llegada a Chile de Rodolfo Lenz, el año 1890, comienzan los estudios científicos de la lengua mapuche. Sus "Estudios Araucanos", publicados en los Anales de la Universidad de Chile, constituyen uno de los aportes cuantitativo y cualitativo más relevantes al conocimiento de esta lengua.

Sin embargo, sólo con la publicación de la reinterpretación fonológica del material contenido en el "Estudio V" de Lenz (realizado por Jorge Suárez, en 1959) se inicia el estudio moderno de la fonética y fonología mapuches.

En nuestro país, el primer lingüista que presenta un estudio exhaustivo de la lengua mapuche es Max S. Echeverría (1964). Doce años más tarde, Adalberto Salas (1976) publica en Estudios Filológicos su "Esbozo fonológico del mapudungun, lengua de los mapuches o araucanos de Chile central". Si se compara este trabajo con los de Suárez y Echeverría se observa que la pauta fonológica propuesta es idéntica en el inventario vocálico, estableciéndose algunas diferencias en la interpretación de ciertos sonidos consonánticos.

Durante la década de los ochenta, dos trabajos sobre la fonología del mapuche destacan por su cobertura y rigurosidad. Estos son "El estrato fónico del mapudungu(n)" (Lagos, 1981), y la "Descripción fonológica del huilliche, un dialecto del mapuche o araucano del centro-sur de Chile" (Álvarez-Santullano, 1986). El trabajo de Lagos, en términos generales, viene a confirmar la existencia de una homogeneidad lingüística entre los hablantes de mapudungun. El trabajo de Álvarez-Santullano, en tanto, establece con claridad el hecho de que el dialecto

\footnotetext{
${ }^{3}$ Una descripción más detallada se encuentra en Salas (1992b).
} 
huilliche es el más divergente respecto del mapuche central y que existe "un alto grado de desmantelamiento del sistema fonológico tradicional (...) reflejo del estado terminal del huilliche osornino" (Salas, 1992b: 509).

Hasta donde sabemos, sólo existen dos trabajos que contienen información respecto de los fonemas segmentales de la lengua hablada por los pehuenches del Alto Bío-Bío. Estos son "Relatos orales en pewenche chileno" (Sánchez, 1989) y "Fonología del pehuenche hablado en el Alto Bío-Bío" (Salamanca, 1997).

\section{METODOLOGÍA DE LA INVESTIGACIÓN}

\subsection{Recolección del material}

\subsubsection{Zona}

Los datos fueron recogidos por Elizabeth Quintrileo, en la comuna de Tirúa, Región del Bío-Bío. Esta comuna costera está situada a 200 kilómetros de Concepción y tiene cerca de 10 mil habitantes, el $70 \%$ de los cuales son mapuches.

\subsubsection{Informantes y entrevistas}

\subsubsection{Informantes}

Para esta investigación se contó con el apoyo de cuatro hablantes adultos, bilingües de mapuche-castellano. Estos hablantes fueron Clementino Lincope, Elena Llevilao, Pedro Huenupil Nahuelpán y Juan Segundo Huenupil Huenupán, los cuales fueron contactados por Valeria Nancupil, coordinadora del proceso de Educación Intercultural Bilingüe en la zona ${ }^{4}$.

\subsubsection{Entrevistas}

El primer viaje a Tirúa se realizó con el fin de conocer a los respectivos asesores y mediante ellos tener acceso a los informantes. En el segundo viaje se realizó la recolección de los datos a través de los cuatro informantes, dos de los cuales pertenecían a la comunidad de Ponotro -lugar ubicado a $1 \mathrm{~km}$ del centro de Tirúa- y los otros dos pertenecientes a la comunidad de Aguas Santas, sector rural de Tirúa, ubicado a $18 \mathrm{~km}$ del centro urbano de Tirúa, junto a la costa. El acceso a este último sector se realizó con vehículo particular, ya que no existían medios de

${ }^{4}$ El Programa de Educación Intercultural Bilingüe es una iniciativa del Ministerio de Educación del Gobierno de Chile que procura que los educandos indígenas se desenvuelvan adecuadamente tanto en su cultura de origen, como en la cultura global. 
transporte que lo vinculasen con el sector urbano. Para llegar al destino específico de los informantes, Elizabeth Quintrileo emprendió una caminata de 1 hora y 45 minutos aproximadamente, ya que el acceso por otra vía no era posible. Una vez allí, entrevistó a Juan Segundo Huenupil, de 70 años de edad. Esta entrevista tomó lugar en la casa del informante, y se prolongó por 1 h y 30 min, aproximadamente.

La siguiente entrevista se realizó a Pedro Huenupil, hermano del informante anterior, quien además es asesor cultural del proyecto intercultural bilingüe. Esta entrevista tomó lugar en la sede social de la comunidad de Aguas Santas y duró aproximadamente 1 h y $45 \mathrm{~min}$.

Al siguiente día se realizaron las dos entrevistas restantes en el sector Ponotro, ubicado a $1 \mathrm{~km}$ del centro de Tirúa. En esta ocasión el acceso a los informantes fue más expedito, ya que existían medios de transporte mucho más accesibles. Así, la primera entrevista de ese día se realizó a Clementino Lincope Llevilao, de 65 años, también asesor cultural del proyecto intercultural bilingüe, además de profesor de mapudungun en las escuelas de Tirúa. Esta entrevista se prolongó por dos horas, ya que él se detenía a dar distintas explicaciones del léxico.

La última entrevista se realizó a Elena Llevilao, de 65 años. Esta entrevista fue un poco más breve que el resto.

Cabe hacer presente, además, que estas entrevistas se realizaron en un ambiente muy cordial, lo cual, sin duda, fue facilitado por el hecho que Elizabeth Quintrileo tiene ascendientes mapuches.

\subsubsection{Lista léxica}

Para facilitar el estudio comparativo de esta variedad del mapuche, se empleó como base la lista léxica que aparece en Salamanca (1997). A esta lista léxica añadimos algunos ítemes que contienen los segmentos críticos que han sido objeto de discordia en la interpretación fonémica realizada en los estudios precedentes. Después de aplicada esta lista, se descartaron aquellos ítemes que no fueron respondidos por los informantes. Así, la lista léxica efectivamente respondida quedó constituida por trescientos ítemes.

Ahora, si bien hubo 300 ítemes que los informantes efectivamente respondieron, dentro de éstos hubo algunos que los informantes pronunciaron con diferencias fónicas tales que no se pudieron atribuir razonablemente a meras variaciones alofónicas o de fluctuación de fonemas, sino a una diferencia en plano léxico concomitante a una diferencia en el plano fónico. No obstante lo anterior, para efectos de esta investigación -que no tiene como foco el nivel léxico- los ítemes en que los informantes no coincidieron fueron igualmente considerados como datos fónicos relevantes, pues, aunque no coincidieran estrictamente en el significado, estaban integrados, sin duda, por fonos pertenecientes al mapuche. 


\section{FONEMAS SEGMENTALES DEL MAPUCHE HABLADO EN TIRÚA}

El marco de referencia escogido para el análisis del material es el propuesto por Pike (1947) y Burquest (2001). La aplicación de este modelo se presenta en Echeverría (1964).

Después de realizado el análisis fonémico de acuerdo con las premisas señaladas por estos autores, hemos podido establecer que el mapuche hablado en Tirúa tiene 24 fonemas segmentales. De ellos, 6 son vocales, 15 son consonantes y 3 son semiconsonantes.

\subsection{Fonemas vocálicos ${ }^{5}$ :}

Los fonemas vocálicos del mapuche hablado en Tirúa son los siguientes: /a, e, i, o, u, î/. La descripción articulatoria de las realizaciones alofónicas de cada uno es la que se presenta a continuación:

\subsubsection{Fonema /a/}

Realización y distribución

Este fonema se realiza como [a]: vocoide baja central no redondeada silábica.

\#

$$
\text { C_C [mári] 'diez' }
$$
\# [vótra] 'barro'

\subsubsection{Fonema /e/}

Este fonema se realiza como [e]: vocoide media anterior no redondeada silábica.

\# [épu] 'dos'

C_C [ailén] 'brasa' __ \# [píúx́e] 'corazón'

\subsubsection{Fonema /i/}

Este fonema se realiza como [i]: vocoide alta anterior no redondeada silábica.

$\#$ [ilón] 'carne'

C_C [lipán] 'brazo' \# [trápi] 'aji'

${ }^{5}$ Las posiciones consideradas para presentar la distribución de los alófonos de las vocales son: posición inicial absoluta (\#_), posición interconsonántica (C_C) y posición final absoluta (_\#). 
El mapuche hablado en Tirúa: Fonemas segmentales, fonotaxis y comparación... / G. SALAMANCA y E. QuintriLEO

\subsubsection{Fonema /o/}

Este fonema se realiza como [o]: vocoide media posterior redondeada silábica.

\# [opún] 'lleno'

C_C [tšoð] 'amarillo' \# [ko] 'agua'

\subsubsection{Fonema $/ \mathrm{u} /$}

Este fonema se realiza como $[\mathrm{u}]$ : vocoide alta posterior redondeada silábica.

\# [unutún] 'mal olor'

C_C [timún] 'arado' \# [kálku] 'brujo'

Hay fluctuación de fonemas entre $/ \mathrm{o} / / \mathrm{u} /$, como se observa en el siguiente ejemplo:

/went ${ }^{\mathrm{r}} \mathrm{u} /$ - /went ${ }^{\mathrm{r} o} /$ 'hombre'.

\subsubsection{Fonema /i/}

Este fonema tiene dos alófonos:

[i]: Vocoide alta posterior no redondeada silábica. Ocurre en posición inicial de palabra.

[ə]: Vocoide media central no redondeada silábica. Ocurre en otros contextos.

En posición final de palabra, variación libre entre [i] y [ə].

\# [ïlmén] 'rico'

$$
\text { \$C_C\$ [móŗ́́e] 'harina }
$$
tostada' \# [pálə] 'mosca'

Hay fluctuación de fonemas entre /ï/ / u/ y /ï/ /e/ como se observa en los siguientes ejemplos:

/rïipï/ - /ŗupu/ 'camino'

/ï̄ï̀m/ -/eñïm/ 'pájaro' 


\subsection{Fonemas consonánticos ${ }^{6}$ :}

Los fonemas consonánticos del mapuche hablado en Tirúa son los siguientes: /p, $\mathrm{t}, \mathrm{k}, \mathrm{v}, \partial, \mathrm{s}, \mathrm{r}, \breve{\mathrm{c}}, \mathrm{t} \mathrm{r}, \mathrm{m}, \mathrm{n}, \tilde{\mathrm{n}}, \mathrm{y}, \mathrm{l}, \lambda /$. La descripción articulatoria de las realizaciones alofónicas de cada uno es la que se presenta a continuación:

\subsubsection{Fonema $/ \mathrm{p} /$}

Este fonema se realiza como [p]: contoide obstruyente oclusiva bilabial sorda oral.

\#___ [payún] 'bigote' V_V [lipán] 'brazo’ *__ \#

Hay fluctuación de fonemas entre $/ \mathrm{p} / \sim / \mathrm{v} /$, como se observa en el siguiente ejemplo:

/pit'un/ /vit'un/ 'humo'.

\subsubsection{Fonema $/ \mathrm{t} /$}

Este fonema tiene dos realizaciones alofónicas en variación libre restringida:

[t] Obstruyente oclusiva alveolar sorda oral.

[t] Obstruyente oclusiva interdental sorda oral.

\#__ [tápəl] - [țápəl]] ‘hoja' V_V [patáka] 'cien’ *__ \#

Decimos que estos fonos manifiestan una variación libre restringida ${ }^{7}$ porque toda vez que aparece el fono interdental, lo hace en variación libre con su correspondiente alveolar; el fono alveolar, en cambio, tiene una distribución considerablemente más amplia, y en la mayoría de los casos no alterna libremente con su contraparte interdental. Así, por ejemplo, [țápəḷ] alterna con [tápəl], pero en [patáka], [t] es siempre alveolar. Esta observación, como veremos, aplica de manera

\footnotetext{
${ }^{6}$ Las posiciones consideradas para presentar la distribución de los alófonos de las consonantes son: posición inicial absoluta (\#_), posición intervocálica $\left(V_{-}\right.$V) y posición final absoluta (_\#).

${ }^{7}$ Llamamos variación libre restringida a un tipo de alternancia que cumple con tres requisitos: a) ocurre entre fonos cuyo estatus de fonemas distintos no se puede demostrar, pues no se presentan en contraste (se distingue así de la fluctuación de fonemas), b) la alternancia libre se restringe a determinados ítemes léxicos (se distingue así de la variación libre a secas) y c) los ítemes léxicos en los que estos fonos alternan libremente son aquellos que en otras variantes dialectales, o en otros momentos históricos, se ha comprobado una relación de contraste.
} 
similar para los alófonos interdentales de los fonemas $/ \mathrm{n} / \mathrm{y} / \mathrm{l} /$.

Cabe hacer notar, además, que este fono presenta una bajísima frecuencia. De hecho, de los fonos interdentales, éste es el que ocurre en menor cantidad de casos.

\subsubsection{Fonema $/ \mathrm{k} /$}

Este fonema tiene dos realizaciones alofónicas en distribución complementaria:

[k] Contoide obstruyente oclusiva velar sorda oral. Ocurre ante vocoide no anterior.

[́́] Contoide obstruyente oclusiva postpalatal sorda oral. Ocurre ante vocoide anterior.

\#__ [katšál] 'hacha' [ḱintún] 'buscar'

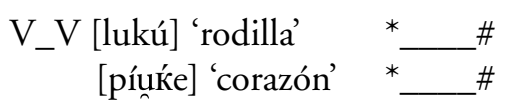

\subsubsection{Fonema $/ \mathrm{t}^{\mathrm{r}} /$}

Este fonema se realiza como [trr]: contoide obstruyente africada alveolar sorda con momento fricativo retroflejo.

$\#$ [trápi] 'ají' V_V [pətŗá] 'guata' * \#

Como se ve, este fonema se realiza fonéticamente como la suma de dos fonos: [t] y [r] . La razón para interpretarlo como un fonema aparte, y no como la suma de los fonemas $/ \mathrm{t} /+/ \mathrm{r} /$, está en la estructura silábica del mapudungun. En efecto, aunque en el inventario de consonantes de esta lengua existen /t/ y /r̦/ y, por lo tanto, no habría necesidad de postular un fonema aparte $/ \mathrm{t}^{\mathrm{r}} /$, la estructura silábica máxima del mapuche es CVC, por lo que si se interpreta [tŗ] como $/ \mathrm{t} /+/ \mathrm{r} / \mathrm{se}$ abre una estructura silábica "anómala" para esta lengua $(\mathrm{CCV})$, lo que sin duda no es una solución fonémica adecuada.

\subsubsection{Fonema / $/ \check{c} /$}

Este fonema tiene dos realizaciones alofónicas en variación libre:

[tš] Contoide africada alveopalatal sorda.

$[\check{s}]$ Contoide fricativa alveopalatal sorda.

\# [tšaðí] 'sal' - [šaðí] 'sal' V_V [ḱétšu] 'cinco'- ['́ééšu] 'cinco' 
RLA. Revista de Lingüística Teórica y Aplicada, 47 (1), I Sem. 2009

Hay fluctuación de fonemas entre $/ \check{c} / / / \mathrm{t} /$ y entre $/ \check{c} / / / s /$, como se observa en los siguientes ejemplos:

/piči/ /piti/ 'pequeño'

/mančun/ /mansun/ 'buey'

Como se observa, en el mapuche hablado en Tirúa ocurre el fono fricativo alveopalatal sordo [š]. La alternancia libre de este fono con su contraparte africado [tš] nos lleva a la interpretación de ambos como alófonos del mismo fonema. Al considerar este fono como alófono del fonema africado, nos aproximamos a la interpretación de Echeverría, aunque nos distanciamos de él en que asignamos estatus fonémico aparte al fono fricativo ápicoalveolar sordo [s].

Por otra parte, la presencia más frecuente de [tš́], tanto en esta variante del mapuche como en las otras donde ambos fonos ocurren -y en la misma distribución-, aconseja la selección de [tš] como la forma básica del fonema.

Por último, resulta conveniente interpretar /č/ como un solo segmento fonémico - y no como la suma de dos segmentos- por dos razones:

a) El patrón silábico máximo en mapuche es CVC y, aún más importante,

b) El fono [š] no tiene estatus fonémico en esta variante del mapudungun, por lo que $/ \mathrm{t} /+/ \check{s} /$ no existe como opción interpretativa con realismo fonémico.

\subsubsection{Fonema /v/}

Este fonema tiene tres realizaciones alofónicas en variación libre:

[v]: Fricativa plana labiodental sonora.

[f]: Fricativa plana labiodental sorda.

$[\beta]$ : Fricativa bilabial sonora.

$\#$

[ $[\mathrm{vu}] \sim[\mathrm{fu}]-[\beta \mathrm{u}]$ 'hilo' V_V[weñevé] -[weñefé] [weñeßé] 'ladrón'
_\#[kuŗév $] \sim[$ kuŗéf $]$ [kurséß] 'viento'

Como se observa, el fonema fricativo labiodental presenta realizaciones sonoras $([\mathrm{v}]-[\beta])$ y sorda $([\mathrm{f}])$, aunque predominan significativamente las realizaciones sonoras. Así, por ejemplo, [léunvu] y [léúnßu] alternan libremente con [léufu], pero los informantes manifiestan clara preferencia por los dos primeros enunciados. Esta es la razón por la cual hemos seleccionado la representación sonora $/ \mathrm{v} /$ como el fonema y no su contraparte sorda. De este modo, el mapuche hablado en Tirúa se distancia de los dialectos del centro-sur, donde, por la razón contraria (predominio de la variante sorda), conviene la selección 
El mapuche hablado en Tirúa: Fonemas segmentales, fonotaxis y comparación... / G. SALAMANCA y E. QUintrileo

del alófono ([f]) como el representante del fonema.

\subsubsection{Fonema /ð/}

Este fonema tiene dos realizaciones alofónicas en variación libre:

[ð]: Fricativa plana interdental sonora.

$[\theta]$ : Fricativa plana interdental sorda.

\# [ $\theta$ añé] -[ðañé] 'nido' V_V [wéða] \# [tšoð] [tšo $\theta]$ 'amarillo' [wé $\theta$ a] 'malo'

En términos de frecuencia, al igual que en el caso del fonema $/ \mathrm{v} /$, el alófono sonoro presenta un claro predominio. Así, por ejemplo, [tšoð] alterna libremente con [tร̌o $\theta$ ], pero el primero de ellos presenta una frecuencia ostensiblemente mayor. Esta es la razón por la cual hemos seleccionado la representación sonora /ð/ como el fonema y no su contraparte sorda.

\subsubsection{Fonema $/ \mathrm{s} /$}

Este fonema se realiza como [s]: fricativa ápicoalveolar sorda oral.

$\#$ [sán.we] 'chancho'

${ }^{*} \mathrm{~V}_{-} \mathrm{V}$

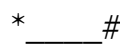

Este es un fonema de muy escasa frecuencia. De hecho, nuestros datos sólo lo reportan en posición inicial.

\subsubsection{Fonema $/ \mathbf{r} /$}

Este fonema tiene dos realizaciones alofónicas en distribución complementaria:

[R]]: Fricativa retrofleja cacuminal sorda. Ocurre en el postmargen silábico.

[r] : Fricativa retrofleja cacuminal sonora. Ocurre en otros contextos.

$\#$ [ŗaḱín] 'bandurria' \# [traŖ] 'pus'

\subsubsection{Fonema $/ \mathrm{m} /$}

Este fonema se realiza como $[\mathrm{m}]$ : nasal bilabial sonora. 
RLA. Revista de Lingüística Teórica y Aplicada, 47 (1), I Sem. 2009

\#__ [mári] 'diez' V_V [nəmái] 'llorar' _ _ [puḱém] 'invierno'

Hay fluctuación de fonemas entre $/ \mathrm{m} / \sim / \mathrm{n} /$, como se observa en el siguiente ejemplo:

/yïn̄ïm/ /yïn̄ïn/ 'hambre’.

\subsubsection{Fonema $/ n /$}

Este fonema presenta tres realizaciones alofónicas:

['n]: Nasal alveolar retrofleja cacuminal sonora.

Ocurre en el postmargen de la sílaba iniciada por consonante retrofleja.

[n]: Nasal interdental sonora.

[n]: Nasal alveolar sonora.

Variación libre restringida entre [n] y [n].

\#__ [namún] 'pie' V_V [vunán] 'podrido' \# [wən] - [wəñ] 'boca'

Al igual que en el caso de $[t]$ y $[\mathrm{t}]$, decimos que $[n]$ y [n] manifiestan una variación libre restringida porque toda vez que aparece el fono interdental, lo hace en variación libre con su correspondiente alveolar; el fono alveolar, en cambio, tiene una distribución considerablemente más amplia, pues no siempre alterna libremente con su contraparte interdental. Así, por ejemplo, [wən] 'boca' alterna libremente con [wə̧, 'boca', pero en [vunán] 'podrido', [n] es siempre alveolar.

Es interesante consignar que en las palabras 'arado' y 'noche' tres de nuestros informantes produjeron consistentemente el fono nasal interdental [n] , es decir, produjeron [timún, y [puṇ], sin alternar libremente con [timún] y [pun]. Sin embargo, uno de los informantes produjo consistentemente contoides alveolares. Al parecer, como veremos en la síntesis comparativa al final de este trabajo, éste es un contraste que está en franco proceso de retirada, del cual sólo quedan débiles huellas en determinados ítemes y en determinadas posiciones.

El alófono retroflejo, por su parte, se observa, por ejemplo, en [motŗîn] 'gordo', donde el fono nasal alveolar se asimila al fono retroflejo [tri], con el cual se inicia la segunda sílaba.

\subsubsection{Fonema $/ \tilde{n} /$}

Este fonema se realiza como [ñ]: nasal mediopalatal sonoro.

\# [ñáwe] 'hija'

$$
\text { V_V [peñí] 'hermano' }
$$
\# [makúñ] 'manta' 
Hay fluctuación de fonemas entre $/ \tilde{n} / \sim / \mathrm{n} /$, como se aprecia en el siguiente ejemplo: /makuñ/ /makun/ 'manta'.

\subsubsection{Fonema $/ \mathbf{y} /$}

Este fonema tiene dos realizaciones alofónicas en distribución complementaria:

[í]: Contoide nasal postpalatal sonora.

Ocurre ante vocal anterior.

[y]: Contoide nasal velar sonora. Ocurre ante vocal no anterior. [yənátui] 'morder'

Hay fluctuación de fonemas entre $/ \mathrm{y} / \sim / \mathrm{n} /$ y entre $/ \mathrm{y} / \sim / \tilde{n} /$, como se aprecia en los siguientes ejemplos:

$$
\begin{aligned}
& \text { /nïpi// /nïpï/ 'ala' } \\
& \text { /ne/-/ñe/ 'ojos' }
\end{aligned}
$$

\subsubsection{Fonema /1/}

Este fonema tiene tres realizaciones alofónicas:

['1]: Lateral alveolar retrofleja cacuminal sonora.

Ocurre ['1] en el postmargen de la sílaba iniciada por consonante retrofleja.

[1]: Lateral interdental sonora.

[1]: Lateral alveolar sonora. Variación libre restringida entre [l] y [l].

La variación libre restringida en este caso se evidencia en que, por ejemplo, [țápəl]] alterna libremente con [tápəl], pero en [wíli], [l] es siempre alveolar y no interdental. Ahora, es interesante observar que uno de nuestros informantes produjo consistentemente la palabra 'carbón' como [kuyúlị], esto es, con interdental. Sin embargo, en los demás informantes la pronunciación más frecuente fue [kuyúl], con lateral alveolar. De ahí que, al igual que como lo señalamos para el caso de $/ \mathrm{n} / \mathrm{y} / \mathrm{t} /$, pensamos que éste es un contraste que está en franco proceso de retirada, del cual sólo quedan huellas débiles. 
El alófono [']], por su parte, se presenta, por ejemplo, en [tṛa'lkán], donde ocurre en el postmargen de la sílaba iniciada por contoide retrofleja ([tṛ]).

\subsubsection{Fonema $/ \lambda /$}

Este fonema se realiza como $[\lambda]$ : contoide lateral mediopalatal sonora.

$\#$

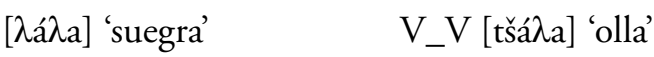
\# [mamád] 'leña'

Hay fluctuación de fonemas entre $/ \lambda / \sim / 1 /$ y $/ \lambda / / / \mathrm{y} /$, como se aprecia en los siguientes ejemplos:

/mamï / /mamïl/ 'leña'

$/ \lambda \mathrm{a} \lambda \mathrm{in} /-/$ yayin/ 'araña'

\subsection{Semiconsonantes}

\subsubsection{Fonema $/ y /$}

Este fonema tiene dos realizaciones alofónicas en distribución complementaria:

Fricativa cóncava alveopalatal sonora $[y]$.

Vocoide muy alta anterior no redondeada [i].

Ocurre $[y]$ cuando se inicia el premargen silábico.

Ocurre [i] en el postmargen silábico.

\# [yaunvén] 'sombra'

V_V [payún] 'bigote' \# [kotšǰri] 'dulce'

\subsubsection{Fonema /w/}

Este fonema tiene dos realizaciones alofónicas en distribución complementaria:

Fricativa velar sonora redondeada [w].

Vocoide muy alta posterior redondeada [u]

Ocurre $[\mathrm{w}]$ cuando está iniciando el premargen. Ocurre $[\mathrm{u}]$ en los otros contextos.

\#___ [wayəlén] 'hombre' V_V [tšawái] 'aro' __ \# [maun] 'lluvia' 


\subsubsection{Fonema $/ \gamma /$}

Este fonema tiene dos alófonos en distribución complementaria:

Fricativa velar sonora no redondeada $[\gamma]$.

Vocoide muy alta posterior no redondeada [i] $]$.

Ocurre $[\gamma]$ en el premargen.

Ocurre [i] en el postmargen.

$\#$

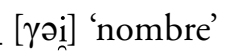
\# [liii] 'blanco'

Como se observa, hemos asignado estatus fonémico al fono fricativo velar sonoro $[\gamma]$. En efecto, consideramos que razones de simetría sistémica aconsejan esta interpretación. Así resultan tres semiconsonantes paralelas a las vocales altas correspondientes:

$\begin{array}{lll}\text { Consonantes / y } & \text { w } & \gamma / \\ \text { Vocales / i } & \text { u } & \text { i } /\end{array}$

\subsection{Cuadros de fonos y fonemas del mapudungun hablado en Tirúa}

Los siguientes cuadros resumen los fonos y fonemas vocálicos y consonánticos del mapudungun hablado en Tirúa:

Tabla I. Fonos vocoides.

\begin{tabular}{|l|l|l|l|l|}
\hline \multicolumn{2}{|l|}{ No red. } & No red. & Redond \\
\hline Muy alta (semiv.) & {$[\mathrm{i}]$} & & {$[\mathrm{i}]$} & {$[\mathrm{u}]$} \\
\hline Alta & {$[\mathrm{i}]$} & & {$[\mathrm{i}]$} & {$[\mathrm{u}]$} \\
\hline Media & {$[\mathrm{e}]$} & {$[\mathrm{]}]$} & & {$[\mathrm{o}]$} \\
\hline Baja & & {$[\mathrm{a}]$} & & \\
\hline & Ant. & Cent. & Posterior & \\
\hline
\end{tabular}


RLA. Revista de Lingüística Teórica y Aplicada, 47 (1), I Sem. 2009

Tabla II. Fonos contoides.

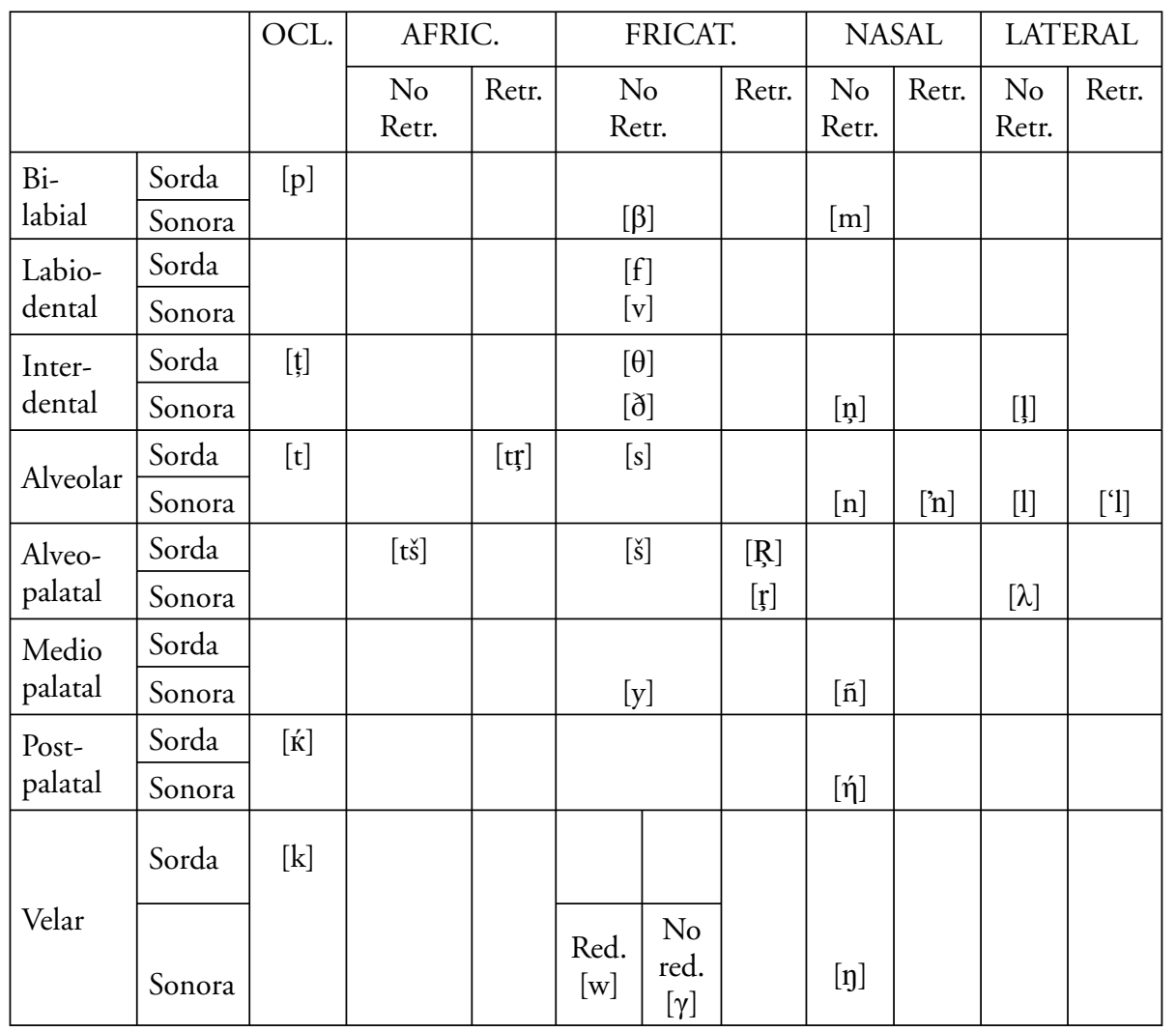

Tabla III. Fonemas vocálicos.

\begin{tabular}{|l|l|l|l|l|}
\hline & \multicolumn{2}{|c|}{ No red. } & \multicolumn{1}{c|}{ No red. } & \multicolumn{1}{c|}{ Redond. } \\
\hline Alta & /i/ & & /i/ & /u/ \\
\hline Media & /e/ & & & $/ \mathrm{o} /$ \\
\hline Baja & & /a/ & & \\
\hline & Ant. & Cent. & Posterior \\
\hline
\end{tabular}


El mapuche hablado en Tirúa: Fonemas segmentales, fonotaxis y comparación... / G. SALAMANCA y E. QUintrileo

Tabla IV. Fonemas consonánticos.

\begin{tabular}{|l|c|c|c|c|c|}
\hline & Labial & Interdental & Alveolar & Palatal & Velar \\
\hline Oclusiva & $/ \mathrm{p} /$ & & $/ \mathrm{t} /$ & & $/ \mathrm{k} /$ \\
\hline Africada & & & $/ \mathrm{t}^{\mathrm{r}}$ & $/ \check{\mathrm{c}} /$ & \\
\hline Fric. Plana & $/ \mathrm{v} /$ & $/ \partial /$ & & & \\
\hline Cóncava & & & $/ \mathrm{s} /$ & & \\
\hline Retrofleja & & & $/ \mathrm{r} /$ & & \\
\hline Nasal & $/ \mathrm{m} /$ & & $/ \mathrm{n} /$ & $/ \tilde{\mathrm{n}} /$ & $/ \mathrm{y} /$ \\
\hline Lateral & & & $/ \mathrm{l} /$ & $/ \lambda /$ & \\
\hline
\end{tabular}

Tabla V. Fonemas semiconsonánticos.

\begin{tabular}{|ccc|}
\hline oblonga & & redondeada \\
\hline $\mathrm{y}$ & $\gamma$ & $\mathrm{w}$ \\
\hline anterior & & posterior \\
\hline
\end{tabular}

\section{FONOTAXIS}

\subsection{Estructura silábica}

Las posibilidades silábicas del mapudungun hablado en Tirúa son las siguientes:

\begin{tabular}{|c|c|c|}
\hline V & /a.ne/ & 'cara' \\
\hline $\mathrm{CV}$ & /ko/ & 'agua' \\
\hline $\mathrm{ScV}$ & /wi.ya/ & 'ayer' \\
\hline VC & /al.ka/ & 'gallo' \\
\hline VSc & 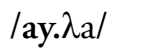 & 'nueve' \\
\hline CVC & /kov.ke/ & 'pan' \\
\hline $\mathrm{ScVC}$ & /ku.yul/ & 'carbón' \\
\hline $\mathrm{ScVSc}_{\mathrm{c}}$ & /ča.way/ & 'aro' \\
\hline
\end{tabular}

En consecuencia, el patrón silábico máximo en el mapuche hablado en Tirúa, al igual que en las otras variantes dialectales de esta lengua, es CVC.

Las restricciones de distribución de acuerdo a clases naturales de segmentos son las siguientes: 
RLA. Revista de Lingüística Teórica y Aplicada, 47 (1), I Sem. 2009

\section{Premargen silábico}

En el premargen o frontis silábico ocurren todas las consonantes, como se observa en los siguientes ejemplos:

Oclusivas

$\begin{array}{lll}/ \mathrm{p} / & \text { /piwke/ } & \text { 'corazón' } \\ / \mathrm{t} / & / \text { tol/ } & \text { 'frente' } \\ / \mathrm{k} / & / \mathrm{kalku} / & \text { 'brujo' }\end{array}$

Africadas

$\begin{array}{lll}/ \mathrm{t}^{\mathrm{r}} / & / \mathrm{t}^{\mathrm{r} a p i} / & \text { 'aji' } \\ / \check{c} / & / \mathrm{c} a \lambda \mathrm{a} / & \text { 'olla' }\end{array}$

Fricativas

Planas

$\begin{array}{lll}\text { /v/ } & \text { /vičayma/ } & \text { 'alto' } \\ \text { /ð/ } & \text { /ðañe/ } & \text { 'nido' }\end{array}$

\section{Cóncava}

/s/ Isañwe/ 'chancho'

Retrofleja

/ŗ / /rïpï/ 'camino'

Nasales

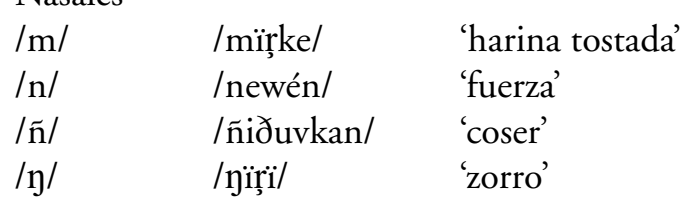

Laterales

$\begin{array}{lll}/ 1 / & / \text { lipay } / & \text { 'brazo' } \\ / \lambda / & / \lambda \mathrm{a} \lambda \mathrm{a} / & \text { 'suegra' }\end{array}$

Semiconsonantes

$\begin{array}{lll}/ \mathrm{y} / & / \mathrm{yu} / & \text { 'nariz' } \\ / \mathrm{w} / & / \text { wilki/ } & \text { 'zorzal' } \\ \mid \gamma / & / \gamma \text { ïy } / & \text { 'nombre' }\end{array}$


El mapuche hablado en Tirúa: Fonemas segmentales, fonotaxis y comparación... / G. SALAMANCA y E. Quintrileo

\section{Postmargen}

De acuerdo a nuestros datos, en el postmargen o coda silábica ocurren los fonemas fricativos (excepto /s/), nasales, laterales y las semiconsonantes; no así los fonemas oclusivos y africados:

Fricativas:

Planas

$\begin{array}{lll}\text { /v/ } & \text { /wilï/ } & \text { 'brillante' } \\ \text { /ð/ } & / \text { čoð/ } & \text { 'amarillo' }\end{array}$

Retrofleja

/ŗ / /pïtrars/ 'piojo (de la ropa)'

Nasales

$\begin{array}{lll}/ \mathrm{m} / & / \text { kuram/ } & \text { 'huevo' } \\ / \mathrm{n} / & \text { /pilun/ } & \text { 'oreja' } \\ / \tilde{\mathrm{n}} / & / \text { makuñ/ } & \text { 'manta' }\end{array}$

/ñ / Imakuñ / 'manta'

Laterales

/l/ $\quad$ /volíl/ $\quad$ 'raíz'

$/ \lambda / \quad$ /mamï $\lambda / \quad$ 'leña'

Semiconsonantes

$\begin{array}{lll}/ \mathrm{y} / & / \text { kïpay/ } & \text { 'venir' } \\ / \mathrm{w} / & / \mathrm{mït}{ }^{\mathrm{r}} \mathrm{Ow} / & \text { 'sacudir' } \\ / \gamma / & / \mathrm{ra} / & \text { 'greda' }\end{array}$

\subsection{Estructura de la palabra}

Las palabras en el mapudungun hablado en Tirúa pueden tener una, dos sílabas o tres sílabas:

Monosilábicas:

/ko/ 'agua'

/li $/$ 'blanco'

Bisilábicas

/we.nïy/ 'amigo'

/ku.yïm/ 'arena' 
RLA. Revista de Lingüística Teórica y Aplicada, 47 (1), I Sem. 2009

Trisilábicas

/wa.yï.len/ 'estrella'

/a.ča.wa入/ 'gallina'

\section{FLUCTUACIONES DE FONEMAS}

Sistematizando los casos de fluctuación de fonemas, tenemos:

Vocales:

$/ \mathrm{u} / \sim / \mathrm{o} /$

/went ${ }^{\mathrm{r}} \mathrm{u} / \sim /$ went $^{\mathrm{r} o} /$ 'hombre'

$\mid \mathrm{i} / \sim / \mathrm{u} /$

/ŗïpï/ / ŗupu/ 'camino'

$\mid \mathrm{i} / / / \mathrm{e} /$

/ïn̄ïm/ /eñïm/ 'pájaro'

Consonantes:

$\mid \mathrm{p} / \sim / \mathrm{v} /$

/pitrun/ /vitrun/ 'humo'

$\mid \check{\mathrm{c}} / \sim / \mathrm{t} /$

/piči/ /piti/ 'pequeño'

$|\check{c} /-/ s|$

/mančun/ /mansun/ 'buey'

$/ \mathrm{m} /-/ \mathrm{n} /$

/yin̄ïm/ /yïñun/ 'hambre'

$/ \tilde{\mathrm{n}} / \sim / \mathrm{n} /$

/makuñ/-/makun/ 'manta'

$/ \mathrm{y} / \sim / \mathrm{n} /$

/nї̈ї/ /nїрї/ 'ala' 
El mapuche hablado en Tirúa: Fonemas segmentales, fonotaxis y comparación... / G. SALAMANCA y E. QuintriLEO

$/ \mathrm{y} / \sim / \tilde{\mathrm{n}} /$

/ye/ /ñel 'ojos'

$\mid \lambda / \sim / 1 /$

/mamï / /mamïl/ 'leña'

$\mid \lambda /-/ y /$

$/ \lambda$ a $\lambda$ in/ $/$ yayin/ 'araña'

\section{CONCLUSIÓN}

Los puntos expuestos anteriormente muestran que no existen grandes diferencias entre la fonología del mapuche hablado en Tirúa y las otras variantes de esta lengua, excepto el huilliche. Esto es claro en el plano de los fonemas segmentales y los rasgos fonotácticos que aquí han sido presentados. Ahora, si se considera la sonoridad de las consonantes fricativas labiodental e interdental, la presencia o ausencia de consonantes interdentales y la presencia o ausencia del fono [̌s] como diferenciadores de variantes dialectales, podemos concluir lo siguiente:

\section{1) Respecto de la sonoridad de las fricativas}

Como hemos dicho, en el mapuche hablado en Tirúa predominan de modo significativo las realizaciones sonoras de las consonantes fricativas labiodental e interdental. De aquí resulta que esta variante se vincula más estrechamente con los grupos mapuches nortinos (grupos I y II de Croese), se distancia levemente del moluche y el pehuenche meridional, y se distancia de manera más pronunciada del huilliche. No obstante, hay que destacar que se trata de un asunto de predominio y no de exclusividad, es decir, no es que las contoides fricativas sordas no se produzcan en absoluto, o que sean rechazadas por los informantes -aunque hay conciencia de que éstas son producidas en mayor proporción en otras zonas mapuches-, sino que ocurren en menor cantidad que sus contrapartes sonoras.

\section{2) Fonos / Fonemas interdentales}

Como se observa en la Tabla IV, en nuestra lista de fonemas no incluimos las consonantes interdentales. Esto se debe a que las hemos considerado realizaciones alofónicas de sus contrapartes alveolares. El argumento que justifica esta interpretación es que en los pocos casos donde estos fonos ocurren, lo hacen en variación libre restringida con sus contrapartes alveolares. Así, por ejemplo, hemos observado ocasionalmente la pronunciación [țəñ], pero este enunciado siempre alterna libremente con [tən], donde [t] y [n] son alveolares. Algo similar sucede con [1]. En efecto, ocasionalmente se observa la pronunciación [țápəḷ], pero siempre en alternancia libre con [tápəl]. 
Ahora bien, hay que destacar que la asignación/no asignación de estatus fonémico a estos fonos en las distintas variantes del mapuche es un asunto de interpretación fonémica siempre controversial (cf. Croese, 1980), por lo que en este punto, al igual que Croese, somos partidarios de revisar la pertinencia de considerar éste como un criterio diferenciador de variedades. Consecuente con esta afirmación, nos parece que en tanto estos fonos se presentan en toda la Araucanía -en mayor o menor proporción- y que su estatus fonémico es un asunto de interpretación sujeto a controversia, se confirma este atributo de homogeneidad al que hemos hecho mención en algunos párrafos precedentes.

3) El fono [s]

La alternancia libre de este fono con su contraparte africado [tš] nos lleva a la interpretación de ambos como alófonos del mismo fonema. Como hemos dicho, al considerar este fono como alófono del fonema africado, nos aproximamos a la interpretación de Suárez (1959) y Echeverría (1964), aunque nos distanciamos de este último en que interpretamos el fono $[\mathrm{s}]$ como un fonema aparte, y no como otra variante del fonema /č/. Ahora, en tanto factor que establece distinciones dialectales, la ocurrencia de este fono en esta variante del mapuche la aproxima al mapuche central y la distancia del dialecto cordillerano nortino (pehuenche hablado en el Alto Bío-Bío) y del dialecto meridional (huilliche), donde estos fonos no ocurren.

Todo lo expresado nos lleva finalmente a respaldar la idea de que existe una apreciable unidad entre todos los dialectos del mapuche, exceptuando el huilliche. En efecto, y como hemos mencionado, tanto en el mapuche central como en el mapuche hablado en Tirúa hay fricativas sordas y sonoras, y en todo este sector ocurren los fonos interdentales, aunque su interpretación fonémica constituye un problema complejo. No incluimos al huilliche, porque en esta variante ocurre una cantidad importante de fenómenos que no ocurren en el resto de la Araucanía: ausencia del fono fricativo $\left[\mathrm{s}^{8}\right]^{8}$; ausencia del fono interdental $[\mathrm{n}]$; presencia de los fonos $[\mathrm{ts}],[\mathrm{x}] \mathrm{y}$ $[\mathrm{h}]$; presencia del fono $[\mathrm{s}]$ como alófono de $/ \theta /$; realización fricativa alveopalatal retrofleja sorda [ş] del fonema /ŗ/; presencia del fono [b] como uno de los alófonos del fonema /w/; estatus fonémico del fono lateral retroflejo ['1]; entre otras diferencias.

En consecuencia, coincidimos plenamente con Salas (1996) en que

La población mapuche actual es sensiblemente uniforme desde el punto de vista lingüístico. Las diferencias en el habla de las distintas regiones son insignificantes: unos cuantos detalles de pronunciación y de vocabulario. El núcleo de la estructura fonológica y gramatical y del vocabulario, es el mismo a través de todo el territorio mapuche (Salas, 1996: 269).

${ }^{8}$ Esta característica, como hemos dicho, también se presenta en el pehuenche hablado en el Alto Bío-Bío. 
El mapuche hablado en Tirúa: Fonemas segmentales, fonotaxis y comparación... / G. SALAMANCA y E. QuintriLEO

\section{REFERENCIAS}

Álvarez-Santullano, Pilar. 1986. Descripción fonológica del huilliche, un dialecto del mapuche o araucano del centro-sur de Chile. Tesis de Magíster en Artes con Mención en Lingüística. Concepción, Chile: Universidad de Concepción.

Burquest, Donald. 2001. Phonological Analysis. A functional approach. Dallas: SIL International.

Croese, Robert. 1980. "Estudio dialectológico del mapuche", en Estudios Filológicos 15, pp. 7-38.

Echeverría, Max. 1964. "Descripción fonológica del mapuche actual”, en Boletín del Instituto de Filología de la Universidad de Chile XVI, pp. 13-59.

Lagos, Daniel. 1981. "El estrato fónico del mapudungu(n)", en Nueva Revista del Pacífico 19-20, pp. 42-66.

Lenz, Rodolfo. 1895-1897. "Estudios Araucanos", en Anales de la Universidad de Chile. Santiago. Tomos XC-XCVIII.

Pike, Kenneth. 1947. Phonemics. A technique for reducing languages to writing. Ann Arbor: The University of Michigan Press.

Quintrileo, Elizabeth y Salamanca, Gastón. 2007. "Fonemas segmentales y muestra lexical del mapuche hablado en Tirúa", en Sociedad Hoy 12, pp. 73-83.

Salamanca, Gastón. 1997. "Fonología del pehuenche hablado en el Alto Bío-Bío", en RLA 35, pp. 113-124.

Salamanca, Gastón. 2001."La lengua de los pehuenches del Alto Bío-Bío: Fonemas segmentales, lista léxica y un cuento tradicional", en Sociedad Hoy 4/5, pp. 9198.

Salas, Adalberto. 1976 "Esbozo fonológico del mapudungun, lengua de los mapuches o araucanos de Chile central", en Estudios Filológicos 11, pp. 143-153.

Salas, Adalberto. 1992a. El mapuche o araucano de Chile. Fonología, gramática y antología de cuentos. Madrid: Fundación MAPFRE AMERICA. Colecciones MAPFRE 1492. Colección Idioma e Iberoamérica.

Salas, Adalberto. 1992b. "Lingüística mapuche. Guía bibliográfica", en Revista Andina 2, pp. 473-537.

Salas, Adalberto. 1996. "Las lenguas indígenas de Chile", en Culturas de Chile. Etnografía. Sociedades Indígenas contemporáneas y su Ideología. Santiago: Andrés Bello.

Sánchez, Gilberto. 1989. "Relatos orales en pewenche chileno", en Anales de la Universidad de Chile. Estudios en honor de Yolando Pino Saavedra. 5ta serie. 17, pp.289360.

Suárez, Jorge. 1959. "The phonemes of an Araucanian dialect", en IJAL 25, pp. 177181. 